\title{
Electrosurgery in dermatosis papulosa nigra: An effective, well-tolerated but less documented tool
}

\author{
Surajit Gorai", Joly Seth, Ayush Bindal, Asit Baran Samanta, Subhas Nag, Bani Kumar \\ Mondal
}

Department of Dermatology, Venereology \& Leprology, Burdwan Medical College \& Hospital, Burdwan, West Bengal, India

\begin{abstract}
Dermatosis papulosa nigra (DPN) is a superficial, black or brown benign papule that develops mainly in dark-skinned individuals over the face and neck. Clinically seborrheic keratosis shows a stuck-on appearance that differentiates it from DPN. Histopathological examination describes that both are identical. Many types of lasers are used as treatments for it. Traditional surgical procedures such as curettage, electrosurgery, and snip/shave excision are still considered as a therapeutic modality. Although electrodesiccation (ED) is a simple procedure, studies on ED use for DPN with a good number of cases are lacking. This paper presents the evaluation safety, efficacy and cost-effectiveness of ED for the treatment of DPN in Fitzpatrick's skin type IV to VI. A total of 40 patients (11 male and 29 female) with DPN over the face and neck with skin type IV-VI were included. Superficial ED (monopolar, low, 2-3.5 W) was done by just touching the lesion under topical anesthesia. All preoperative photographs as well as 2-, 4- and 8-week post-procedure photographs were examined by two independent dermatologists who had no prior information concerning the procedure, (both known hereafter as "blinded observer"): one for examining efficacy in terms of poor improvement $(0 \%-25 \%)$, fair $(26 \%-50 \%)$, good $(51 \%-75 \%)$, or excellent $(76 \%-100 \%)$, by counting completely cleared lesions; and the other for examining side effects in terms of hypo/hyperpigmentation and scarring. As a result, $85 \%$ of patients showed excellent improvement (>75\% clearance), $7.5 \%$ good (51\%-75\% clearance), $5 \%$ fair $(26 \%-50 \%$ clearance) and $2 \%$ poor ( $0-25 \%$ clearance) as measured by the "blinded" observer. Post-inflammatory hyperpigmentation was observed in $15 \%$ cases, whereas hypo-pigmentation was documented in $7.5 \%$ cases. Only in two cases (5\%) was lesional scarring noted. Those complications were cleared after eight weeks. In this study, ED was observed to be an effective, well-tolerated and cost-effective method for treatment of DPN in colored skin. Complications were few, and therapy was performed appropriately with effective postoperative measures.
\end{abstract}

Keywords: dermatosis papulosa nigra (DPN); electrodesiccation; colored skin

Citation: Gorai S, Seth J, Bindal A, Samanta AB, Nag S, et al. Electrosurgery in dermatosis papulosa nigra: An effective, well-tolerated but less documented tool. J Surg Dermatol 2020; 5(1): 26; http://dx.doi.org/10.18282/jsd.v1.i2.26.

* Correspondence to: Surajit Gorai, Department of Dermatology, Venereology \& Leprology, Burdwan Medical College \& Hospital, Burdwan, West Bengal, India; drsgorai@gmail.com

Received: $25^{\text {th }}$ June 2019; Accepted: $27^{\text {th }}$ September 2019; Published Online: $15^{\text {th }}$ October 2019

\section{Introduction}

Dermatosis papulosa nigra (DPN) is a superficial, black or brown, smooth surfaced, benign papule that develops mainly in dark-skinned individuals. They grow in size and number, yet individual lesions remain small, mainly

Copyright (C) 2020 Gorai S, et al. This is an Open Access article distributed under the terms of the Creative Commons Attribution-Non Commercial 4.0 International License (http:// creativecommons.org/licenses/by-nc/4.0/), permitting all non-commercial use, distribution, and reproduction in any medium, provided the original work is properly cited. 
affecting the face and neck region. Treatment is indicated mainly for aesthetics. Sometimes larger lesions may raise suspicions of untoward complications but proper counselling regarding a benign course will suffice in most of the cases. Spontaneous regression of lesion is rare. Clinically seborrheic keratosis shows a stuck-on appearance that differentiates it from DPN. However, histopathologically they are identical. Many experts consider DPN to be a variant of seborrheic keratoses ${ }^{[1]}$.

A few studies have documented lasers as treatment for seborrheic keratoses ${ }^{[2]}$. So, different lasers such as pulse dye treatment (PDL), potassium titanyl phosphate (KTP), and neodymium-doped yttrium aluminium garnet (Nd:YAG) are used for the treatment of DPN with varying success ${ }^{[3]}$. Though lasers are the recent trend, traditional surgical procedures such as curettage, electrosurgery and snip/shave excision are still considered as therapeutic modalities. Post-inflammatory pigmentary complications are the primary concern in colored skin. Electrodesiccation (ED) is a very simple, efficacious, and low-cost modality used in dermatosurgery. Many diseases such as verucca, molluscum, milia, epidermal nevi, skin tags, pyogenic granuloma, etc., can be treated effectively by $\mathrm{ED}^{[4]}$. However, this modality is less documented for DPN treatment as a single effective therapy. ED also serves as a therapeutic modality for seborrheic keratoses even though studies in DPN lacked significant number of cases. The aim of the present study was to examine the safety, efficacy and cost-effectiveness of this age-old ED procedure for the treatment of DPN in Fitzpatrick's skin type IV to VI.

\section{Materials and methods}

A total of 40 patients with DPN on the face and neck with skin type IV-VI were recruited from the outpatient department for this prospective, non-randomized and double-blind study. It was carried out from Jan 2015 till June 2015. Willing patients of over 18 years old, with skin type IV-VI and clinically having more than four DPN lesions, were recruited after obtaining informed consent. Patients who received prior treatment for DPN, patients with cardiac pacemaker, and pregnant ladies were excluded from the study. After taking pre-procedure photograph, EMLA (2.5\% lignocaine $+2.5 \%$ prilocaine) was applied under occlusion for 50-60 min. Then, with the angled fine tip probe, superficial ED (monopolar, low, $2-3.5 \mathrm{~W}$ ) was performed by just touching the lesions momentarily, considering grayish white discoloration as the end point. The patients who volunteered were observed. After treatment, each of them was asked about the prevalence of pain and burning sensations. They were also examined for erythema and edema. In four cases (where lesions were extensively distributed over the whole face and neck), all the lesions were treated in a single sitting. Postoperatively, all the patients were prescribed with topical fusidic acid and hydrocortisone cream for a period of seven days. Additional chemical (30 SPF) sunscreen was prescribed for four weeks. After the second week, monthly follow-up sessions were done on the patients for three months. Photographs were taken in each follow-up session. All preoperative photographs as well as 2-week, 4-week and 8-week post-procedure photographs were examined by two independent dermatologists who had no prior information concerning the procedure or patient selection, (both known hereafter as "blinded observer") to rate the efficacy and side effects. Efficacy was analyzed as poor improvement $(0-25 \%)$, fair $(26 \%-50 \%)$, good $(51 \%-75 \%)$, or excellent $(76 \%-100 \%)$ by counting completely cleared lesions ${ }^{[5]}$. Side effects were examined in terms of hypo/hyperpigmentation and scarring.

\section{Results}

A total of 40 patients (11 male and 29 female) were included in the study. All of them are Indians who reside in West Bengal. The age distribution was 19-62 years old. Gender distribution according to skin types is stated in Table 1. Each patient was assessed for the total number of lesions, with photographs taken preoperation and two weeks postoperation. Rate of efficacy, as measured in terms of complete clearance of lesions, was expressed in percentage. Incomplete clearance of lesion was regarded as non-clearance. $85 \%$ of patients showed excellent improvement (> 75\% clearance), $7.5 \%$ good $(51 \%-75 \%$ clearance), $5 \%$ fair (26\%-50\% clearance) and $2 \%$ poor ( $0-25 \%$ clearance) as measured by the "blinded observer" (Figure 1). In the 'excellent' group, 58.8\% demonstrated $100 \%$ clearance of lesion. In the immediate postoperative period, there was localized erythema (85\%) and edema (70\%) over the ED sites (Figure 2). Figure 3 showed the left side of the face and neck of a patient preoperatively. Figure 4 showed excellent result postoperatively. 3 patients $(7.5 \%)$ complained of mild pain during the procedure and therefore reapplication of topical anesthetic agent for another 15 mins was required. 4 (10\%) patients reported burning sensation instantly after the procedure.

Photographs at two weeks post-procedure and monthly for three months thereafter were evaluated by the "blinded observer" for documentation of complications. Post-inflammatory hyperpigmentation was observed in $15 \%$ cases and hypopigmentation was documented 
in $7.5 \%$ cases. In 2 cases (5\%), lesional scarring was observed (Figure 5). One of the patients had an extensive lesion that required a second sitting for total removal. After eight weeks of pigmentary changes and scarring, the results were not perceptible.

Table 1. Skin types with sex distribution

\begin{tabular}{ccc}
\hline SKIN TYPES & Male & Female \\
\hline IV & 3 & 11 \\
V & 6 & 14 \\
VI & 2 & 4 \\
Total & 11 & 29 \\
\hline
\end{tabular}

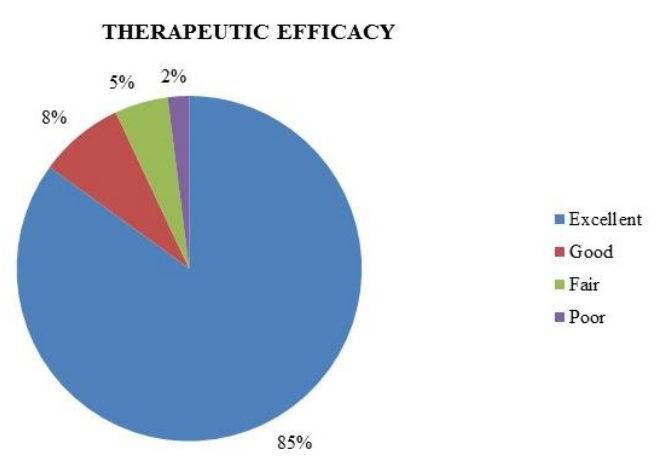

Figure 1. Therapeutic efficacy, two weeks after treatment

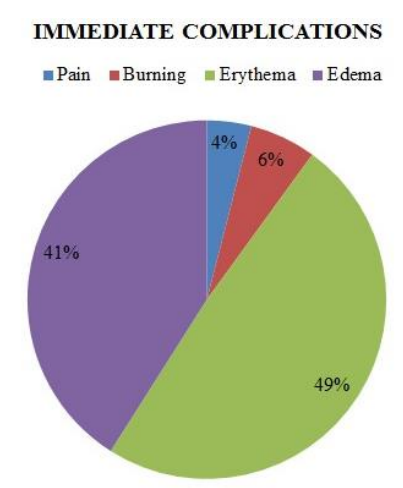

Figure 2. Immediate complications after treatment

\section{Discussion}

Our study revealed that ED is safe, well tolerated and efficacious in removing DPN painlessly by using topical anesthesia under occlusion for an adequate duration. Studies with quantitative assessment of the number of lesion clearances well established its efficacy. In a rando-

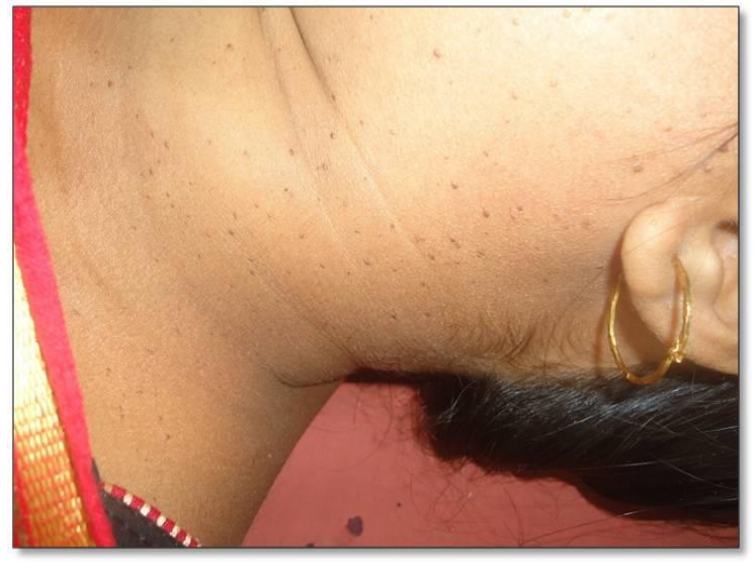

Figure 3. Pre-operative photograph showing extensive lesions

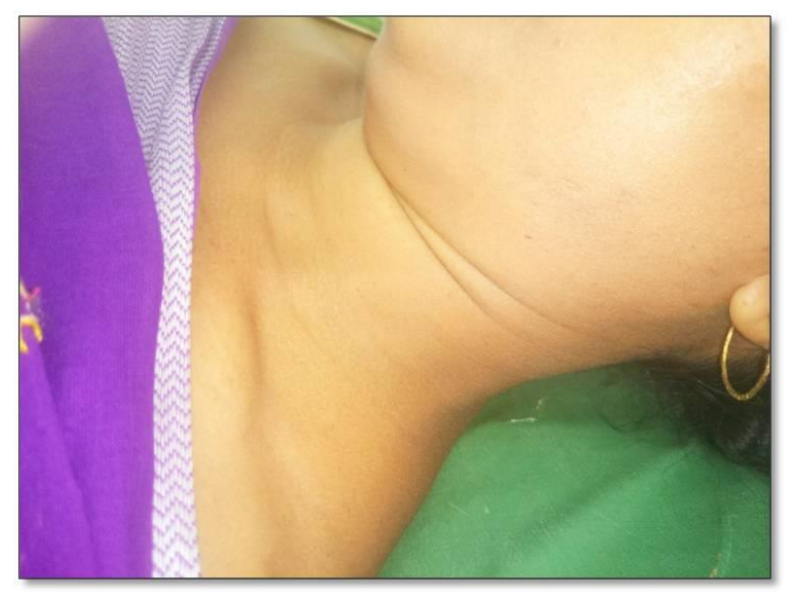

Figure 4. Postoperative photograph showing almost no lesion

mized control trial of ED, curettage and PDL by Garcia and colleagues showed that there was no significant difference in clinical efficacy among these three procedures. In their study, one "blinded observer" evaluated and recorded the findings from pre- and post-procedure photographs. "Blinded observers" performed a global assessment without quantification by lesional count. The assessment reported that patients favored ED over others, including PDL, due to lesser pain. In the same study, patients preferred ED with best cosmetic outcome followed by curettage and laser ${ }^{[6]}$. Niang and team carried out a prospective study on 30 DPN patients in Senegal. The analysis of results over a period of six months demonstrated ED as a perfect therapeutic modality ${ }^{[7]}$.

Multiple expensive and high technology lasers were capturing the market gradually for different cosmetic and dermatosurgical conditions. Certain lasers other than PDL have been in trials in DPN; however, successful techniques with lasers were not as convincing compared to trusted age-old ED. Kundu et al. compared ED with 


\section{Late complications}

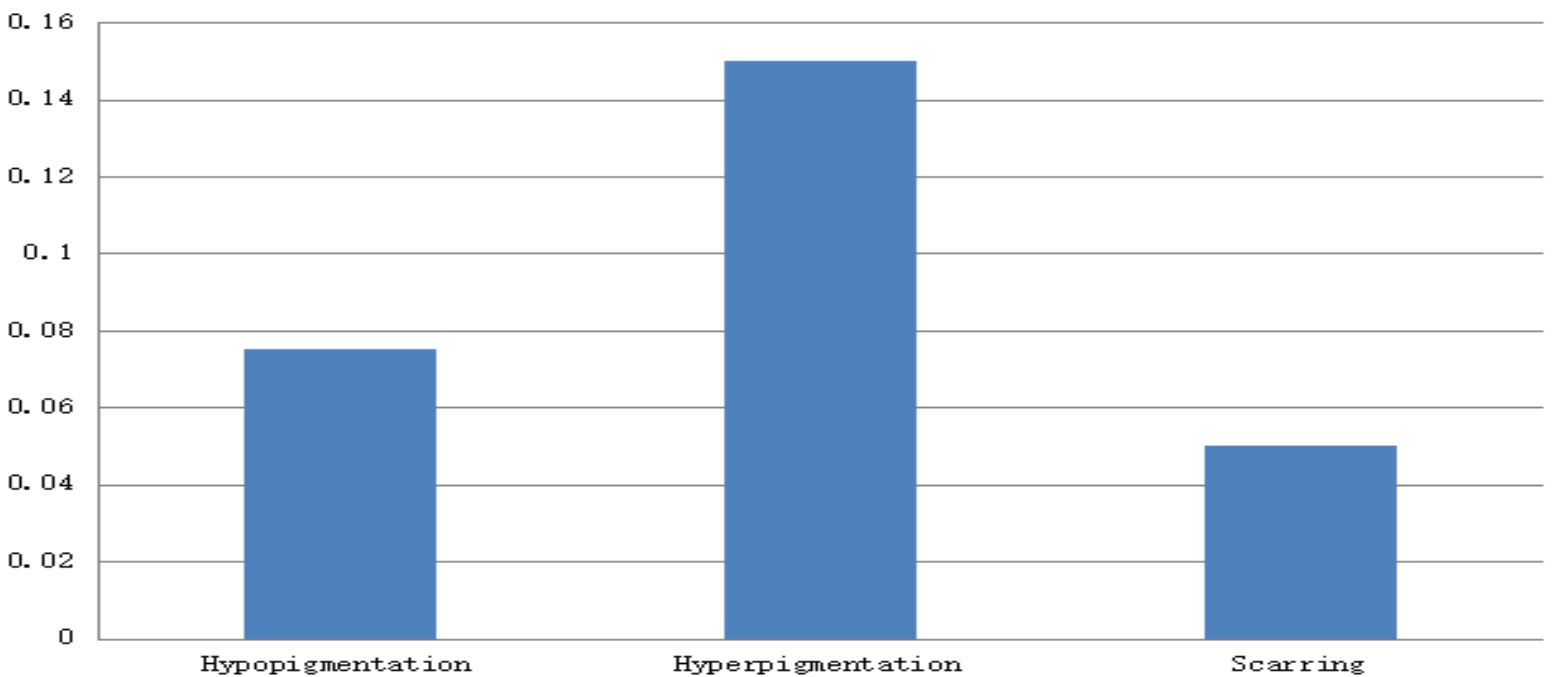

Figure 5. Late complications

KTP laser for DPN in 14 subjects (skin type IV to VI) with a split-face model and the study demonstrated that in terms of efficacy, the ED and KTP lasers had no significant difference. The analysis showed that a small number of patients experienced discomfort with KTP laser as compared to ED, in the event of both cases having no administration of anesthesia in the study proto$\mathrm{col}^{[5]}$.

In darker skin types, the most detrimental side effect is post-inflammatory pigment alteration. In the case of DPN patients who seek it exclusively for cosmetic concerns, post-inflammatory pigment alteration usually affects patient satisfaction. In our study, post-inflammatory hyperpigmentation was observed only in a small number of cases after two weeks, and it would disappear after 8-12 weeks with minimal therapy. In contrast, a study by Gracia MS et al. reported a high rate $(50 \%)$ of post-inflammatory hyperpigmentation ${ }^{[6]}$. These were due to the removal of each lesion after ED by a gauge piece for depth assessment, as per study protocol. The better outcome in terms of pigmentary complication in our study was explained by the accurate depth assessment and the dried residue being left as it was after reaching the end point without wiping it out. Along with that, we prescribed post-procedural topical antibiotic and least potent steroid combination for a certain period of days with sunscreen applied, which contributed to the result ${ }^{[5]}$.

Minor side effect profile was observed in darker skin type. We encountered no recurrence after three months of follow-up sessions. Immediate postoperative lesional mild erythema and edema disappeared within 3-5 days afterward. A few cases of post-inflammatory hypopig- mentation were also observed after a week, which thereafter healed within 4-6 weeks. Minimal scarring that was perceived in two cases disappeared within eight weeks. ED targets each lesion separately, and care should be taken for it to be kept superficial in order to avoid post-inflammatory hyperpigmentation. This method requires time compared to laser. In the case of multiple lesions, more than one sitting may be needed ${ }^{[8]}$. Katz et $a l$. discussed erbium-doped $1550 \mathrm{~nm}$ fractionated laser as an effective tool for DPN though it was based on a single patient evaluated by a "blinded observer". The improvement rate of $75 \%$ was based on global assessment instead of lesion count ${ }^{[9]}$. Schweiger described an improvement of $70 \%-90 \%$ with long-pulsed Nd:YAG in two patients. However, the assessment method was not described ${ }^{[3]}$.

The presented study was restricted due to requirement of long-term follow-up session and the process of juxtaposition with other modalities. Further research is suggested to distinguish this method for treatment modalities. Cost-effective measures optimized for Indian scenario should be evaluated. ED machines are simple, cheap and affordable. In contrast, lasers have higher cost and limited availability at specific centers. Treatment outcome of DPN with a laser is inadequate. Hereby, we have shown that a simple ED is an effective method for treating a condition such as DPN. The procedure was judicious, and with appropriate postoperative measurement, complications were able to be reduced.

\section{Conclusion}

The current study observed ED to be an effective, 
well-tolerated and cost-effective method for the treatment of DPN in colored skin. Complications are minimal if the therapy procedure is performed accurately with effective postoperative measurement.

\section{Conflict of interest}

The authors declared no potential conflict of interest with respect to the research, authorship, and/or publication of this article.

\section{References}

1. James WD, Berger T, Elston D. Epidermal nevi, neoplasms and cysts. $11^{\text {th }}$ ed. In: James W, Berger T, Elston D, (editors). Andrews's diseases of the skin: Clinical dermatology. Philadelphia: Elsevier; 2011. p. 626.

2. Pierson D, Bandel C, Ehrig T. Benign epidermal tumors and proliferations. $2^{\text {nd }}$ ed. In: Bolognia J, Jorizzo JL, Rapini RP (editors). Dermatology. New York: Mosby; 2003. p. 1717.

3. Report of two cases. J Cosmet Laser Ther 2008; 10(2): 120-122. doi: 10.1080/14764170801950070.

4. Sebben JE. The hazards of electrosurgery. J Am Acad Dermatol 1987; 16: 869-872. doi: 10.1016/S0190- 96
22(87)80221-9.

5. Kundu RV, Joshi SS, Suh KY, Boone SL, Huggins RH, et al. Comparison of electrodesiccation and potassium-titanyl-phosphate laser for treatment of dermatosis papulosa nigra. Dermatol Surg 2009; 35: 1079-1083. doi: 10.1111/j.1524-4725.2009.01186.x.

6. Garcia MS, Azari R, Eisen DB. Treatment of dermatosis papulosa nigra in 10 patients: A comparison trial of electrodesiccation, pulsed dye laser, and curettage. Dermatol Surg 2010; 36: 1968-1972. doi: 10.1111/j.1524-4725.2010.01769.x.

7. Niang SO, Kane A, Diallo M, Choutah F, Dieng MT, et al. Dermatosispapulosanigra in Dakar, Senegal. Int J Dermatol 2007; 46(Suppl 1): 45-47.

8. Karadag AS, Ozkanli S, Mansuroglu C, Ozlu E, Zemheri E. Effectiveness of the pulse dye laser treatment in a Caucasian woman with dermatosis papulosa nigra. Indian J Dermatol 2015; 60: 321. doi: 10.4103/00 19-5154.156447.

9. Katz TM, Goldberg LH, Friedman PM. Dermatosis papulosa nigra treatment with fractional photothermolysis. Dermatol Surg 2009; 35: 1840-1843. doi: 10.1111/j.1524-4725.2009.01302.x. 